\title{
ROLE OF EDTA ON METAL REMOVAL FROM REFINERY WASTE CATALYSTS
}

\author{
MEENA MARAFI \& MOHAN S. RANA \\ Petroleum Research Center, Kuwait Institute for Scientific Research, Kuwait
}

\begin{abstract}
Waste spent hydro-processing catalysts contains significant quantities of heavy metals such as Mo, Ni, $\mathrm{V}$, and Co. The disposal of waste refinery catalysts is a serious environmental concern because of the presence of heavy metals. Hence, an attempt has been made to recover the metals from a refinery waste ARDS catalyst using organic acids such acetic acid, oxalic acid, citric acid, and ethylenediaminetetraacetic acid (EDTA) as leaching agents. EDTA has been found to be a most reactive agent for extraction along with the recycling of the leachant. EDTA acts as an auxiliary complexing agent and binds selectively to the metals particularly Ni, Co, V, and Mo. Hence EDTA is the most active complexing agent for the selective binding of metals in a variety of matrixes. The effect of different process conditions such as $\mathrm{pH}$, temperature $\left(35-60^{\circ} \mathrm{C}\right)$, concentration $(2-10 \mathrm{wt} . \%)$, reaction time (1-6 h), and solid to liquid ratio $(1: 15-1: 40)$ on metal recovery was investigated. It was found that the EDTA can remove $97 \%$ of Mo, $95 \%$ of $\mathrm{Ni}$, and $94 \%$ of $\mathrm{V}$ under optimum process conditions. Subsequently, the effluents of the process are also separated as metal salts, solvent, and EDTA.

Keywords: waste catalyst, deactivation, hydroprocessing, spent catalyst.
\end{abstract}

\section{INTRODUCTION}

Supported catalysts are primarily used in the petroleum refinery to improve the quality of fuel. The catalyst gets deactivated with time, and at the end of the run it becomes "waste catalyst." In a typical commercial atmospheric residue hydrodesulfurization (ARDS) process, the hydroprocessing catalysts are discarded when their performance drop below an acceptable level (i.e. 0.5 wt.\%). These discarded or spent catalysts are known as waste catalysts. In recent years, large quantities of spent hydroprocessing catalysts are discarded as solid wastes from the petroleum refining industries [1]. These waste catalysts are hazardous, and their disposal in the landfills is not advised mainly due to the heavy metal leaching in the soil [2], [3]. Worldwide, about 840,000 t/year spent catalysts is generated, which is expected to further increase with the heavier feedstock [4], [5]. Despite the huge amount of waste, their disposal processes have not been widely developed from the point of view of environmental and economic importance [6]-[8]. On the other hand, the cost and demand for metals (Mo, $\mathrm{V}, \mathrm{Ni}$, and $\mathrm{Co}$ ) have been growing significantly, which, must be recovered. Apart from the landfilling spent catalyst that can be used in road and cement industries; they are neither environmentally clean nor economically beneficial [7], [8]. Thus, the need for separating high-value metals and catalyst support required further studies. Some other methods require high energy input and further generate environmental concerns [3]. However, mineral acids (or basic) have the disadvantage of being corrosive and require special handling and storage, and expensive materials for the equipment [1], [9]. Alternatively, organic acids can form soluble complexes with metal ions and can be extracted under mild conditions [10].

In general, $\mathrm{Co}$ or $\mathrm{Ni}$ is particularly delicate metal to remove from hydro-processing spent catalysts by conventional aqueous leaching techniques where aqueous leach solution of ammonia and an ammonium salt remove the limited content of cobalt present in spent catalyst [2], [3]. Alternatively, using a complexing agent, it expected that $\mathrm{Co}(\mathrm{Ni})$ is the easiest metal to be recovered with very high purity. The $\mathrm{Ni}(\mathrm{Co})$-EDTA complex are well reported in the literature, which is soluble in basic $\mathrm{pH}$ [11]. 
The metals leaching with organic chelating agents do not require high temperatures, and some of them can be reused in the process [12], [13]. Due to its recoverable nature, this process is more economical than any other process. Moreover, it is eco-friendly, noncorrosive, has an easy operational methodology, and most importantly produce no hazardous byproducts [1]. The high efficiency of metal extraction and moderate thermodynamic stabilities of the metal complexes make this process more favorable compared to others. The EDTA leaching method is based on the principles that form metal complexes, where the complex nature will depend on the chemical operating parameters of the solution [14]. Authors have studied EDTA as a source of leaching agent and found that $\mathrm{pH}$ plays an essential role to stabilize and destabilize the metal complexes [14]. EDTA is expected to have an affinity to interact with transition metal atoms via its organic ligands to their transit electrons of the $d$ and $f$ orbits (M, i.e. Co, Ni, Mo, and $\mathrm{V}$ ), which is specific to its complex formation at various $\mathrm{pH}$. [8], [9]. Moreover, due to the commercial importance of EDTA, its (chelant) reuse and conservation remain the second objective of this study.

In the present study, hydroprocessing spent catalyst metal recovery process was investigated using organic leaching agents, which have significant leaching efficiency towards surface metal such as $\mathrm{Mo}, \mathrm{Ni}, \mathrm{V}$, and Al. Thus, the objective is to assess the metals removal efficiency of organic acids (acetic acid, oxalic acid, citric acid, and EDTA) at different operating conditions such as the type of chelating agent, aging time, solid-to-liquid ratio, agitation (magnetic stirring and ultrasonic), and temperature. Maximum metals were extracted with the EDTA chelating agents while lowest extraction was observed with acetic acid. Even though EDTA has shown to be a potential chelating agent for metal (Mo, Ni, and V) removal, not many studies are available on the influences of the operating conditions. Therefore, more attention has been paid to the EDTA based chelating agent and its operating conditions.

\section{EXPERIMENTAL}

Refinery waste spent catalysts were collected from KNPC's commercial ARDS unit. The spent catalyst is de-oil, grind $(<500 \mu \mathrm{m})$ and decoked. The powdered spent catalyst was mixed with the selected organic reagent and agitated under required operating conditions.

\subsection{Characterization of spent catalyst}

The chemical composition of spent and the decoked catalysts were evaluated using inductively coupled plasma (ICP) analysis, where the required amount of solid spent catalyst was treated in concentrated hot $\mathrm{HCl}$ and $\mathrm{HNO}_{3}$ acids in a 1:3 ratio. Subsequently, the digested mixture was cooled and filtered to perform ICP analysis, which is shown in Table 1. The textural properties of the fresh and spent catalyst were determined using BET method (Table 1).

Table 1: Elemental composition and textural properties of spent catalysts.

\begin{tabular}{|l|c|c|c|c|c|c|c|}
\hline \multirow{2}{*}{ Catalyst } & \multicolumn{4}{|c|}{ Composition, wt.\% } & \multicolumn{3}{c|}{ Textural properties } \\
\cline { 2 - 8 } & $\mathrm{Mo}$ & $\mathrm{Ni}$ & $\mathrm{V}$ & Carbon & $\mathrm{SSA}, \mathrm{m}^{2} / \mathrm{g}$ & $\mathrm{TPV}, \mathrm{ml} / \mathrm{g}$ & $\mathrm{APD}, \mathrm{nm}$ \\
\hline Fresh & 8.0 & 3.3 & - & - & 252 & 0.58 & 16.2 \\
\hline Spent & 7.5 & 4.8 & 9.8 & 18.1 & 102 & 0.05 & 8.7 \\
\hline Decoked & 6.73 & 4.3 & 10.0 & $<1$ & 235 & 0.51 & 13.0 \\
\hline
\end{tabular}


The qualitative analyses of deposited species (metals and carbon) were carried out by SEM EDX, which is shown in Fig. 1(a) and (b). The dispersive energy spectrum is displayed in digitalized form with the $\mathrm{x}$-axis representing X-ray energy and a relative account of elemental intensity. The characteristic X-ray emission spectra indicated by reference line $(\mathrm{K} \alpha)$ to the energy level diagram, in which horizontal line (Fig. 1(b)) represents the binding energy of the $\mathrm{K}$ shell or subshell. The carbon content in spent catalysts was further analyzed by using thermogravimetry analysis (TGA) of the temperature range of room temperature to $850^{\circ} \mathrm{C}$ (Fig. 1(c)).

\subsection{Extraction procedure (acid or complexing agents)}

The stepwise description of metal recovery from spent hydroprocessing catalyst is shown in Fig. 2, which contains various systematic steps during the physical and chemical treatments. The process has easy applicability to ensure about $95 \%$ recovery of all metals (Mo, V, and $\mathrm{Ni}$ a at high purity. Usually, the catalyst is unloaded from the reactor, which contains physically adsorbed impurities that are mostly hydrocarbon. These contaminants were removed by de-oiling with naphtha and then extracted with toluene (hot washing) using Soxhlet extraction to remove the residual oil. The dried catalyst extrudates were crushed and ground to fine powder (using ball-mill) and then sieved to a fine particle of spent catalyst $(<500 \mu \mathrm{m})$. This catalyst contains coke (soft and hard coke) and deposited metals (Ni and $\mathrm{V}, \mathrm{Fe}$, etc.). The carbon species (coke) is removed by a de-coking method using robust conditions particularly temperature of $600^{\circ} \mathrm{C}$, air of $50 \mathrm{~mL} / \mathrm{min}$ and treatment time of 12 hours. The de-coking spent catalyst powder was treated with aqueous leaching agents (organic acids: EDTA, citric acid, oxalic acid, and acetic acid) solution with or without ultrasonic agitation. The treated mixture was filtered and washed in which dissolved species (mainly metals V, Ni and Mo and aluminum in traces) can be separated as a liquid solution while un-dissolved (i.e. alumina) remains as a residue. EDTA salt is partially soluble in water, but its solubility increases with increasing $\mathrm{pH}$ of the solution. Hence all the experiments were carried out within the $\mathrm{pH}$ range of 10.2-10.4. The filtrate of the solution was treated with $\mathrm{HNO}_{3}$ and $\mathrm{pH}$ was kept at 1.5 to precipitate EDTA while metals remained in the aqueous solution.
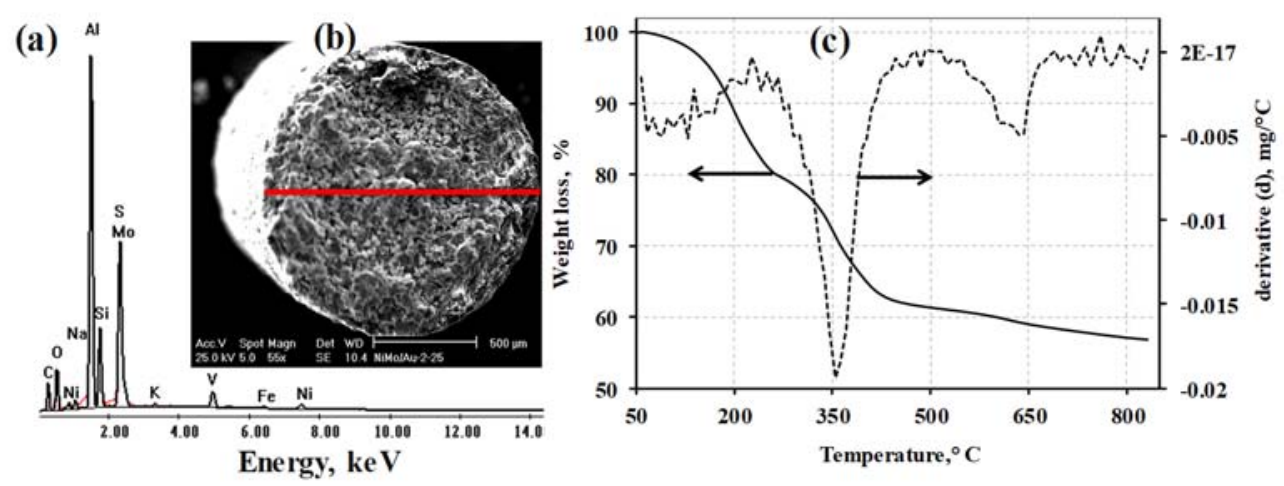

Figure 1: Identification of deposited metal on the spent catalyst. (a) EDS spectrum of spent catalyst; (b) SEM micrograph; and (c) TGA profile of deposited carbon. 


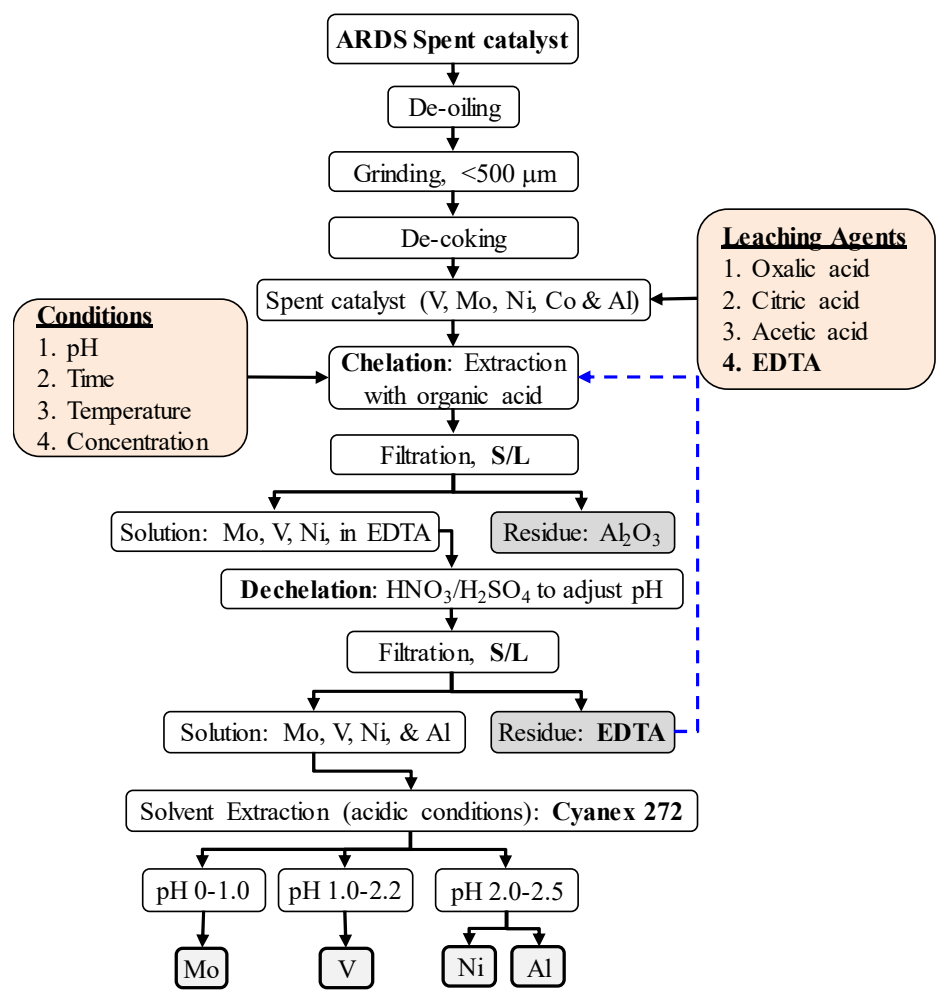

Figure 2: A simplified conceptual schematic flow diagram of the metal recovery process for spent hydroprocessing catalyst.

\subsubsection{Solvent extraction procedure}

The metals in solution were further separated by using solvent extraction using Cyanex 272 in 1:1 ratio of solute and solvent respectively at different $\mathrm{pH}$. The equilibrium $\mathrm{pH}$ of the aqueous phase varied in the range 0 to $6 \mathrm{pH}$, as shown in Fig. 2. The solvent extraction was carried out in a batch by shaking equal volumes $(25 \mathrm{ml})$ of the aqueous solution of the extracted metals with an organic solvent (20\% Cyanex 272 in white spirit) using ultrasonic water bath at $40^{\circ} \mathrm{C}$ to attain equilibrium, which is about $5 \mathrm{~h}$. The aqueous and organic phases were then divided using a separating funnel.

\section{RESULTS AND DISCUSSION}

Refinery ARDS spent catalysts were investigated for their physicochemical characterization to understand composition as well as textural properties. Spent catalyst was microporous, and deactivated species were identified deposited on the surface of the catalysts particularly around the pore mouth [15]-[17]. Deposited metal species were identified with SEM-EDS analysis (Fig. 1(a) and (b)), while carbon content was analyzed with the help of thermogravimetric analysis (Fig. 1(c)). Spent catalyst was de-oil (washed with naphtha), ground and de-coked in order to remove carbon content and subsequently enhance the metal leaching. Table 1 presents an account of textural properties are given where spent catalyst showed a decrease in specific surface area (SSA) $60 \%$, total pore volume (TPV) $85 \%$, and average pore diameter (APD) $46 \%$, which were recovered after de-coking $90 \%, 87 \%$, and 
$80 \%$, respectively. These results indicated that significant pores were blocked due to the carbon deposition. The decoking results also infer to the efficient leaching process where decoked catalyst metals are exposed on the surface as well to allow leaching agent to reach internal pores. Hence efficient decoking process is expected to attribute to the better masstransfer from solid to liquid during the leaching process. Usually, deposited surface metals (also known as catalytic sites) are separated in an aqueous medium by selective removal from the bulk material (support). The deposited metals (Mo, V, Ni, and Co) have a wide range of oxidation $(+\mathrm{Ve})$ state, which has a higher affinity to form coordinate bonds with organic ligands. However, the electrostatic interaction between the transition metal ion (variable oxidation state) and the presence of lone pairs of an electron on the ligands (chelating agents) enhance the interaction and as a results higher metal extraction. The inorganic acid or base treatments have been studied in detail [1], [6], but the separation of metal remains low; thus, in this study, four organic acids were used to remove metals. Operating parameters such as temperature, ultrasonic shaking, time, acid concentration, and the solid/liquid ratio on metal leaching were investigated.

\subsection{The chelate effect}

The solvation (interaction of solute and solvent) factor in organic solvents is less significant than in aqueous solution (hydration); therefore, the entropy change of chelation in an organic solvent will be more significant than that in an aqueous solution. The polar organic molecules are adsorbed on the catalyst surfaces from aqueous solutions mainly due to the hydrophobic nature of transition metal ions toward hydration or the stronger attraction due to the coordinative electron pair of the organic ligands. The lower the solubility of an organic molecule, the more strongly it is adsorbed. An effect of various organic acids (chelating agents) with and without ultrasonic agitation is shown in Fig. 3. A significant effect has been observed for the ultrasonic agitation, which gives high energy to vibrate grinding catalyst powder along with a chelating agent that allows more collision to occur thereby increasing the rate of solvation. However, ultrasonic vibration is also selectively better for some specific metals, which further indicated the different type of interaction between the metal and a chelating agent.

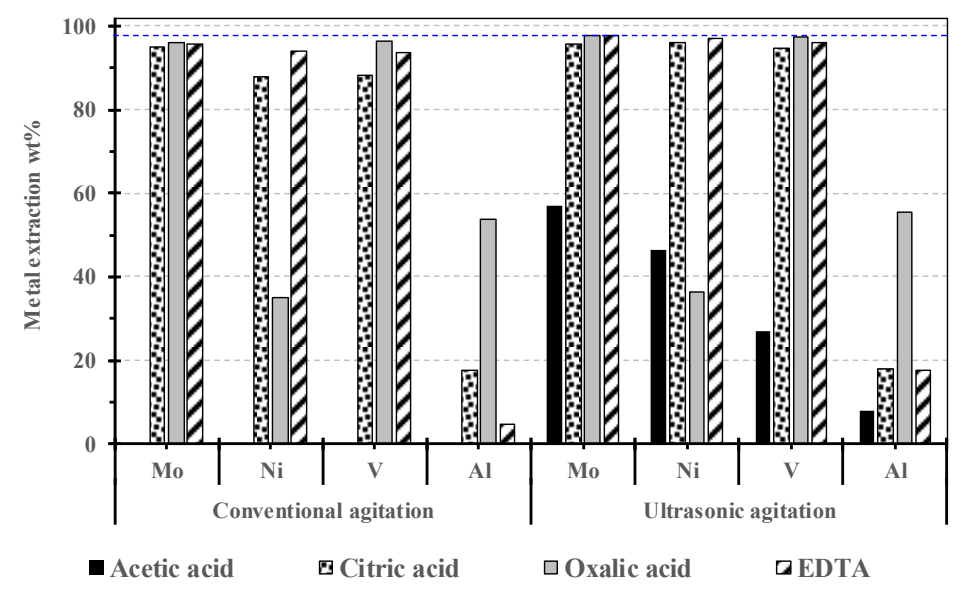

Figure 3: Role of ultrasonic agitation on metal extraction efficiency using various organic acids for metal extraction from spent catalyst. 
Acetic acid showed moderate extraction of metals while oxalic acid is highly effective for Mo and V extraction. Further extraction condition changes could lead only to a slight increase in the removal of these two metals with oxalic acid. With citric acid, a substantial increase was seen in the amounts of $\mathrm{V}, \mathrm{Ni}$ and Mo extraction, and the extraction percentages of these metals exceeded $95 \%$ at $50^{\circ} \mathrm{C}$. High metal leaching results indicated that best equilibrium was achieved with EDTA. During the process, EDTA also can be recovered after the elimination of metal, which is shown in Fig. 4.

Fig. 5 showed the effect of temperature on different chelating agents, which indicated noticeable results for oxalic acid and EDTA for Mo and V while citric acid shows slightly lower metal elimination, particularly $35^{\circ} \mathrm{C}$. Oxalic acid showed significantly low elimination of $\mathrm{Ni}$, which may correspond to the oxalate surface complexation in the presence of water. The facile hydration may prevent dissociation of Ni from spent catalyst.

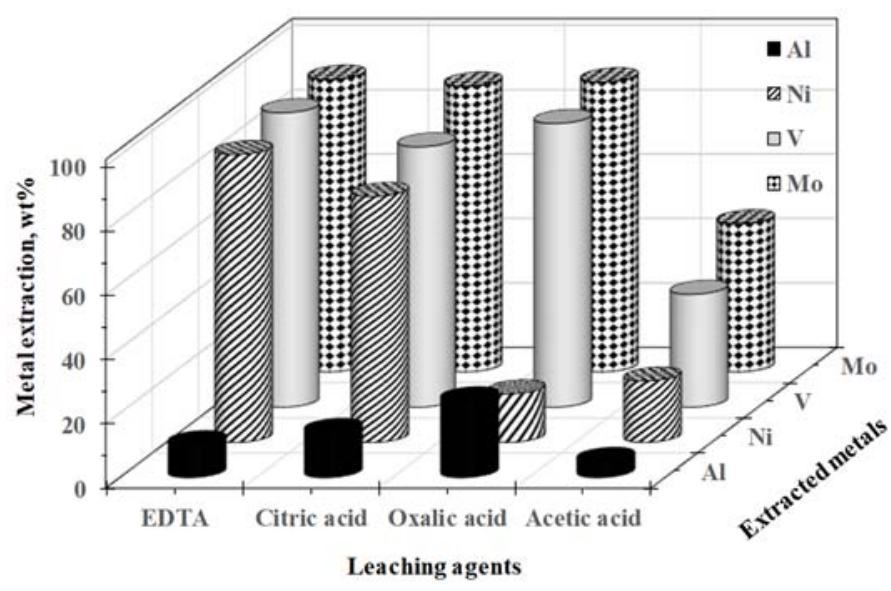

Figure 4: Effect of leaching agent at $10 \mathrm{wt} . \%, 35^{\circ} \mathrm{C}, \mathrm{S} / \mathrm{L}$ ratio $1: 40$, and leaching time $6 \mathrm{~h}$.

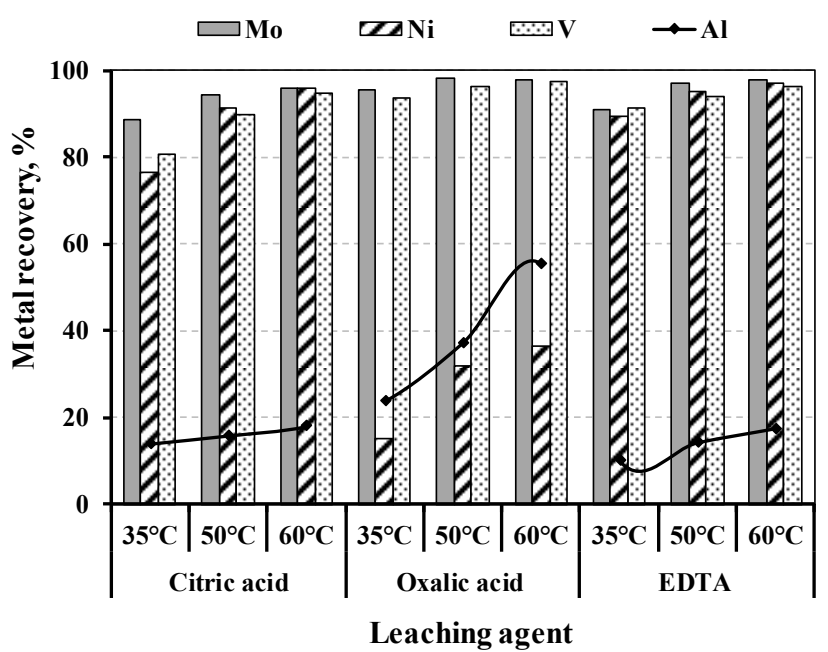

Figure 5: Effect of temperature with various leaching agents at $60^{\circ} \mathrm{C}$ and $6 \mathrm{~h}$ aging time. 


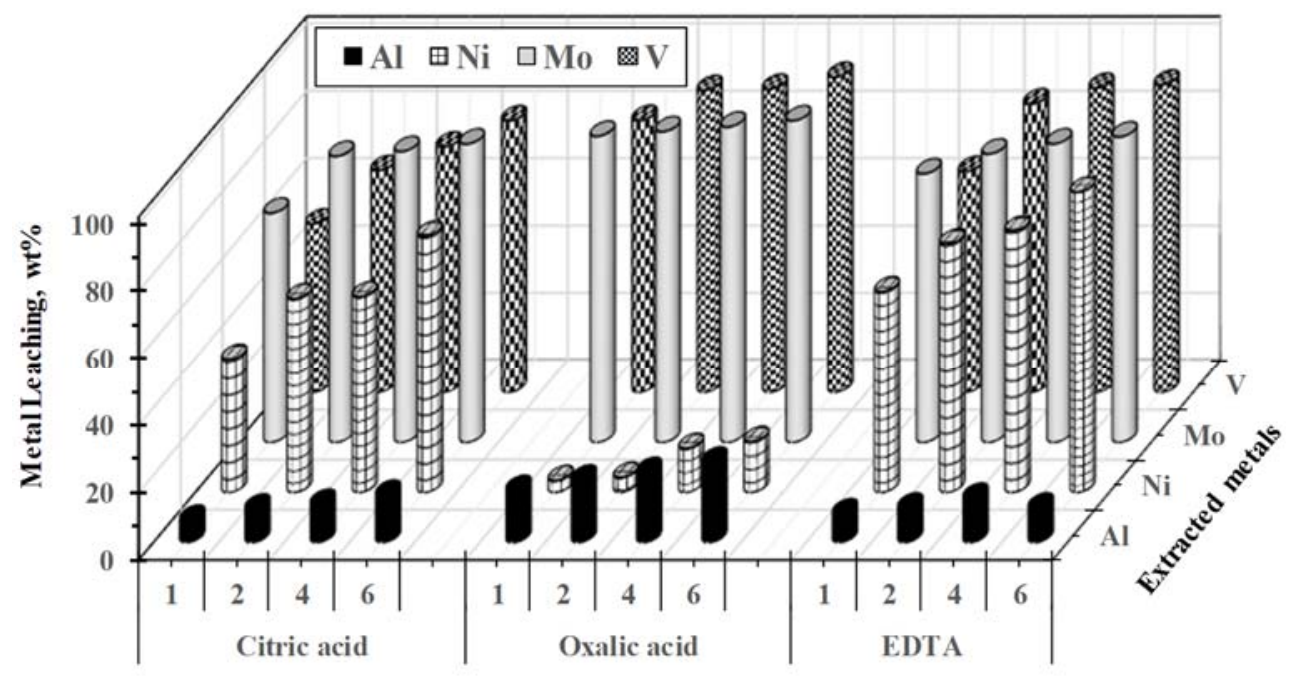

Leaching agents and time, $h$

Figure 6: Effect of EDTA leaching time metals recovery $\left(50^{\circ} \mathrm{C}\right.$, solid/liquid ratio 1:40, and $10 \mathrm{wt} . \%$ EDTA concentration).

Among the organic acids tested for leaching of metals from spent hydro-processing catalysts, EDTA exhibited the highest activity in the range of $96-98 \%$ extraction of all three metals (Mo, V, and Ni). Among organic acids, both oxalic acid and EDTA were slightly more effective than citric acid for Mo extraction. Extraction of Mo was achieved at 97-98\% with both oxalic acid and EDTA; whereas, with citric acid, Mo recovery was around 95\%. For Mo extraction, the effectiveness of leaching agents are ranked in the following order: EDTA = oxalic acid $>$ citric acid $>$ acetic acid. EDTA exhibited high activity for the leaching of all three significant metals (Mo, V, and Ni) with around $90 \%$ extraction even at a low temperature of $35^{\circ} \mathrm{C}$. An increased temperature from 35 to $60^{\circ} \mathrm{C}$ significantly improved the leaching, and more than $96 \%$ of all three metals were leached at $60^{\circ} \mathrm{C}$. To assess the aging effect on the metal extraction with EDTA as a function of time, the EDTA was very active at the initial stage of time where significant extractions of the three metals (Mo, V, and Ni) occurred, as shown in Fig. 6. A probable reason for this observation is EDTA adsorption capacity, which showed the high removal of Mo (97.8\%) > Ni $(97 \%) \geq \mathrm{V}(96 \%)$ after $6 \mathrm{~h}$ time on stream and at $50^{\circ} \mathrm{C}$. The role of different organic acids can be explained based on their $\mathrm{Ka}$ (acid dissociation constant) values, which is a good indicator of the degree of ionization. The lower the Ka value, the higher is the acid strength and the smaller the conjugated base produced by the acid [2], [3].

Usually, EDTA chelation process takes place at high $\mathrm{pH}$, i.e. in the range of 10.2 to 10.4 , where the reaction between ligands and cation takes place within an equilibrium reaction time of $6 \mathrm{hrs}$. The interaction of chelating agent on the catalyst surface metal is shown in eqn (1) [11], [12];

$$
[\text { EDTA (En) }]^{-2}+\mathrm{M}^{+2} \rightarrow[\text { Chelate (En)-M], }
$$

where $\mathrm{M}$ is cation while EDTA act as a ligand and complex formation that depends on the vulnerable surface dominated. The amount of extracted metals is calculated as the ratio of 
metals present in the aqueous solution to form a complex in the spent catalyst. The removal efficiency of the EDTA agents was increased with an increase in chelating agent concentration, as shown in Fig. 7.

This study intends to elaborate the variety (concentration) of the surface interfacial chemistry involved between deposited metal on alumina and the leaching agent, which showed optimum concentration at about 8 to 10 weight percent of the total weight of the catalyst used for extraction. The presence of more than one metal ion leads to different formation constants of the leaching solvent such as metal-EDTA complexes. The separation of metals from the de-chelated filtrate at various equilibrium $\mathrm{pH}$ by using solvent extraction, and the individual metal separation is shown in Fig. 8.

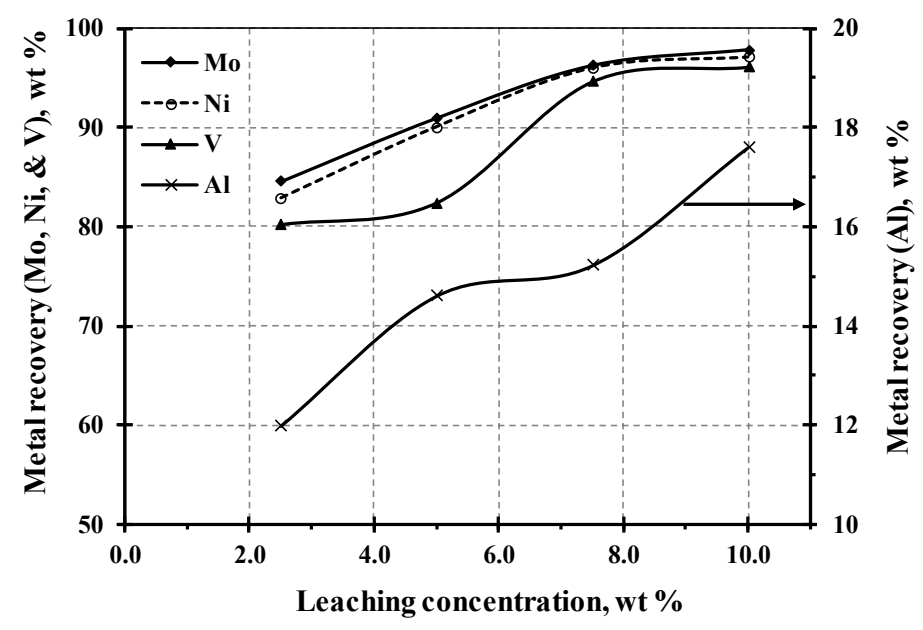

Figure 7: Effect of EDTA leaching concentration at $60^{\circ} \mathrm{C}, 6 \mathrm{~h}$, and solid/liquid ratio=1:40.

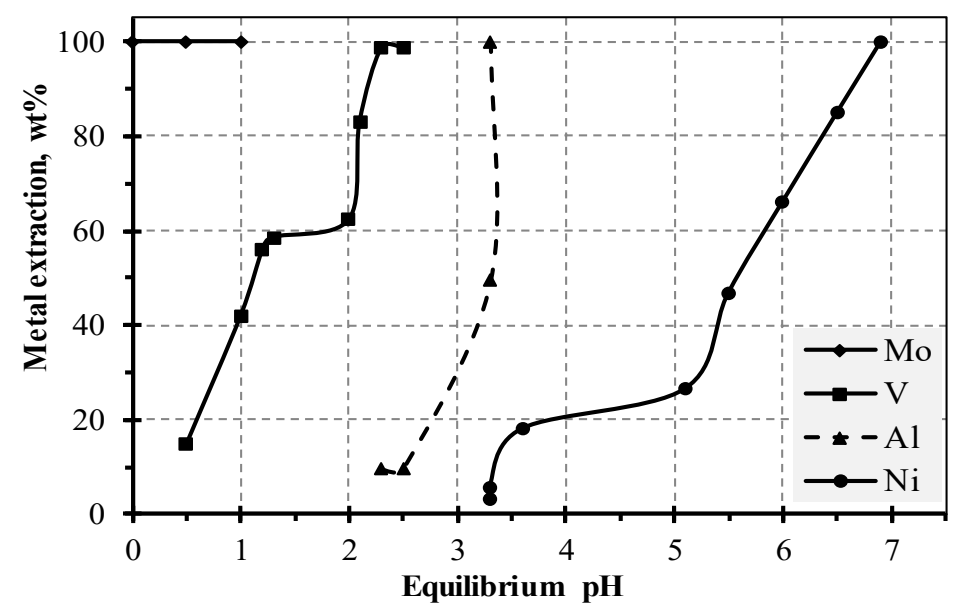

Figure 8: Extraction of metals using Cyanex 272 at different $\mathrm{pH}$. 
Before the separation of the individual metals ( $\mathrm{Mo}, \mathrm{V}, \mathrm{Ni}$, and $\mathrm{Al}$ ) by solvent extraction, the EDTA-metal complex was destabilized, and EDTA was precipitated using concentrated nitric acid and removed by filtration. The EDTA reagent was separated and recovered as a solid residue with high purity from the extracted solution. To extract the EDTA, a mixture of the solution was treated with nitric acid with the controlled $\mathrm{pH}$ at 1.2 where EDTA was precipitated. The precipitated EDTA was filtered, washed, and dried at room temperature. The solid amount of EDTA recovery was greater than $95 \mathrm{wt} . \%$. The purity of EDTA was tested using chemical analysis, which showed traces of $\mathrm{Ni}(100 \mathrm{ppm})$ and $\mathrm{Al}(400 \mathrm{ppm})$.

EDTA is widely used a high-cost complexing agent. Therefore, to make this process economically feasible, dechalation (EDTA recycling) process was developed where metals are present as EDTA-metal complex [Chelate (En)-M], which was decomposed in the presence of acid $\left(\mathrm{H}_{2} \mathrm{SO}_{4}\right.$ or $\left.\mathrm{HNO}_{3}\right)$, where EDTA was precipitated and separated as solid with more than $95 \mathrm{wt} . \%$. The dechalation reaction is shown in eqn (2) [14],

$$
\text { [Chelate (En)-M] }+\mathrm{H}_{2} \mathrm{SO}_{4} \rightarrow \mathrm{MSO}_{4} \cdot 6 \mathrm{H}_{2} \mathrm{O}+\text { Chelating agent (solid). }
$$

The de-chelation process is influenced by EDTA solubilization and strongly depends on the $\mathrm{pH}$. The reaction is acid catalyzed where dissolution of metals favors EDTA recovery at low $\mathrm{pH}$. An increase in $\mathrm{pH}$, the solid phase is decreasing mainly due to the equilibrium where hydrolysis is favored over complexation. Hence the dechelation is evidenced only for EDTA at the most acidic conditions since EDTA weaker complexing ability makes it less able to prevent hydrolysis.

EDTA can be recovered by lowering the $\mathrm{pH}$ to 2 and precipitate as $\mathrm{H}_{4}$ EDTA and reintroducing into the process. The use of recovered EDTA in the process has economic advantages and avoids its presence in wastewaters, which is associated with environmental concerns. EDTA is a chelating agent widely used in chemical and agriculture industries. The recovery of EDTA from washing effluents is essential to reduce the cost of EDTA and prevent the outlet into the environment. Thus, a method of EDTA recycling has been proposed. The $\mathrm{pH}$ of the resulting liquor is then adjusted, causing the precipitation of EDTA in acidic and alkaline solutions, in the form of $\mathrm{H}_{4}$ EDTA and $\mathrm{Na}_{2} \mathrm{H}_{2}$ EDTA, respectively. The recycled solid EDTA can be further purified as a pure compound. However, the filtrate liquid is treated as required to permit environmental disposal. The EDTA containing liquor can also acidify to a pH of about 0.5 to 2 using $\mathrm{H}_{2} \mathrm{SO}_{4}$. Selection of acid mainly depends on desirable specific salt formations produced during the precipitation of the EDTA, in its acid form (Hacid EDTA) at room temperature. The yield of EDTA is likely to increase with decreasing temperature most preferably at $0^{\circ} \mathrm{C}$.

\section{CONCLUSION}

The discarded hydroprocessing spent catalysts are known as waste catalysts, the disposal of which is a serious environmental concern. EDTA has shown to be a successful chelating agent for the extraction of metals from spent catalysts. The chemistry of leaching depends on the solvation properties of mixed solution composition and operating conditions. Various independent operating variables $\left(6 \mathrm{~h}\right.$ aging, $60^{\circ} \mathrm{C}$, and $1: 40$ solid/liquid ratio) are tested to extract deposited metals more than $95 \%$. Oxalic acid exhibited the highest leaching efficiency toward Mo and V metals but feeble recovery for Ni. The efficiencies of leaching agent EDTA showed the higher capacity to extract metals with a comparison to other organic acids such as citric acid, oxalic acid, and acetic acid. EDTA acts as a supplementary complexing agent and binds selectively surface metals (Ni, Co and V, and Mo). EDTA was established to be highly effective in metal removal, and about $97 \%$ of Mo, $95 \%$ of $\mathrm{Ni}$ and $94 \%$ of $\mathrm{V}$ were removed under optimum process conditions. The effectiveness of ultrasonic agitation showed 
significant improvement in the leaching of metals with that of mechanical stirring. The effluents of the process such as solvent and EDTA are also separated with the yield of greater than $95 \%$.

\section{ACKNOWLEDGMENT}

The authors would like to thank the Kuwait Foundation for the Advancement of Sciences (KFAS) for its financial support (project: PF037C).

\section{REFERENCES}

[1] Marafi, M., Stanislaus, A. \& Furimsky, E., Handbook of Spent Hydroprocessing Catalysts: Regeneration, Rejuvenation, Reclamation, Environment and Safety, 2nd ed., Elsevier, 2017.

[2] Marafi, M. \& Stanislaus, A., Spent hydroprocessing catalyst management: A review. Part II. Advances in metal recovery and safe disposal methods. Resources, Conservation and Recycling, 53(1-2), pp. 1-26, 2008.

DOI: 10.1016/j.resconrec.2008.08.005.

[3] Al-Sheeha, H., Marafi, M., Raghavan, V. \& Rana, M.S., Recycling and recovery routes for spent hydroprocessing catalyst waste. Industrial and Engineering Chemistry Research, 52(36), pp. 12794-12801, 2013. DOI: 10.1021/ie4019148.

[4] Marafi, M. \& Rana, M.S., Refinery waste: The spent hydroprocessing catalyst and its recycling options. WIT Transactions on Ecology and the Environment, 202, pp. 219230, 2016.

[5] Rana, M.S., Vinoba, M. \& AlHumaidan, F.S., Sustainability challenges in oil and gas development in the Middle East and North Africa. Current Sustainable/Renewable Energy Reports, 4, pp. 232-244, 2017.

[6] Marafi, M. \& Rana, M.S., Refining waste spent hydroprocessing catalyst and their metal recovery. World Academy of Science Engineering and Technology, 11, pp. 893897, 2017.

[7] Marafi, M. \& Rana, M.S., Metal leaching from refinery waste hydroprocessing catalyst. Journal of Environmental Science and Health, Part A, 53(11), pp. 9517-9519, 2018. DOI: $10.1080 / 10934529.2018 .1470802$.

[8] Marafi, M. \& Stanislaus, A., Spent catalyst waste management: A review. Part IDevelopments in hydroprocessing catalyst waste reduction and use. Resources, Conservation and Recycling, 52, pp. 859-873, 2008.

[9] Marafi, M., Rana, M.S., Navvamani, R. \& Al-Sheeha, H., Utilization of waste spent hydroprocessing catalyst: Development of a process for full recovery of deposited metals and alumina support. WIT Transactions on Ecology and the Environment, vol 163, WIT Press: Southampton and Boston, pp. 237-249, 2012.

[10] Marafi, M. \& Furimsky, E., Selection of organic agents for reclamation of metals from spent hydroprocessing catalysts. Erdoel Erdgas Kohle, 121, pp. 93-96, 2005.

[11] Goel, S., Pant, K.K. \& Nigam, K.D.P., Extraction of nickel from spent catalyst using fresh and recovered EDTA. Journal of Hazardous Materials, 171(1-3), pp. 1-3, 2009. DOI: 10.1016/j.jhazmat.2009.05.131.

[12] Chauhan, G., Pant, K.K. \& Nigam, K.D.P., Chelation technology: A promising green approach for resource management and waste minimization. Environmental Science and Processes Impacts, 17(1), pp. 12-40, 2015. DOI: 10.1039/c4em00559g. 
[13] Chauhan, G., Pant, K.K. \& Nigam, K.D.P., Conceptual mechanism and kinetic studies of chelating agent assisted metal extraction process from spent catalyst. Journal of Industrial and Engineering Chemistry, 27, pp. 373-383, 2015. DOI: 10.1016/j.jiec.2015.01.017.

[14] Chauhan, G., Pant, K.K. \& Nigam, K.D.P., Extraction of nickel from spent catalyst using biodegradable chelating agent EDDS. Industrial and Engineering Chemistry Research, 51(31), pp. 10354-10363, 2012. DOI: 10.1021/ie300580v.

[15] Ancheyta, J., Rana, M.S. \& Furimsky, E., Hydroprocessing of heavy petroleum feeds: Tutorial. Catalysis Today, 109(1-4), pp. 3-15, 2005.

DOI: 10.1016/j.cattod.2005.08.025.

[16] Leyva, C., Rana, M.S., Trejo, F. \& Ancheyta, J., NiMo supported acidic catalysts for heavy oil hydroprocessing. Catalysis Today, 141(1-2), pp. 168-175, 2009. DOI: 10.1016/j.cattod.2008.03.030.

[17] Rana, M.S., Ancheyta, J., Sahoo, S.K. \& Rayo, P., Carbon and metal deposition during the hydroprocessing of Maya crude oil. Catalysis Today, 220-222, pp. 97-105, 2014. DOI: 10.1016/j.cattod.2013.09.030. 